\title{
CARACTERÍSTICAS CLÍNICAS DE LA ROTURA HEPATICA RELACIONADA CON LA PREECLAMPSIA SEVERA EN EL INSTITUTO NACIONAL MATERNO PERINATAL
}

\author{
Ronald Meza Salcedo ${ }^{1}$, Walter Jerry De la Peña Meniz' ${ }^{1,2,4}$, Alberto Martin Díaz Seminario ${ }^{1,3,5}$, \\ Hernán Sandoval Manrique ${ }^{1}$, Peter Pool Málaga Bustamente ${ }^{1}$, Julio César Cano Loayza ${ }^{1}$, \\ Marco Antonio Delgado Fuentes ${ }^{1}$, Carla Calsina Acuña ${ }^{1}$, Christian Cachay Alor ${ }^{1}$, \\ Kenny Eli Villalobos Corrales', Héctor Ramos Bravo', Isaías Magallanes Zuzunaga', \\ Franklin Mendoza Torres ${ }^{1}$
}

\begin{abstract}
RESUMEN
La rotura hepática del embarazo (RHE) es una patología de baja incidencia pero de alta morbimortalidad, siendo una complicación devastadora en pacientes con preeclampsia severa (PES); se reportan aproximadamente 200 casos en la literatura mundial y algunos casos individuales en la literatura nacional. Objetivos: El objetivo principal fue describir las características clínicas más frecuentes en pacientes con diagnóstico de PES admitidas a la Unidad de Cuidados Intensivos Materna (UCIM) del Instituto Nacional Materno Perinatal (INMP). Materiales y métodos: Se revisaron las historias clínicas de pacientes con el diagnóstico de RHE atendidas en la UCIM del INMP en el período comprendido entre 2004 y 2013; se utilizó una ficha de recolección de datos y se realizó un análisis de frecuencias para los signos y síntomas más comunes. Resultados: El 92,6\% de casos (25/26) de RHE se presentó en PES; la estancia hospitalaria fue de 19,4 $\pm 13,24$ días (2-

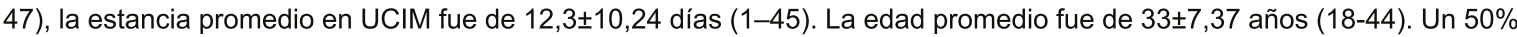
$(10 / 20)$ de casos se presentó en gestantes pretérmino y un $30 \%(6 / 20)$ en puerperas. El $65 \%(13 / 20)$ de las pacientes eran multíparas; el 38,1\% (8/21) fallecieron. Las características clínicas más frecuentes fueron: taquicardia materna $(61,9 \%)$, dolor abdominal $(57,1 \%)$, hipotensión arterial $(57,1 \%)$, palidez $(52,4 \%)$, distensión abdominal $(47,6 \%)$, hipertensión arterial $(38,1 \%)$. Conclusiones: La RHE es una complicación infrecuente de la PES en el INMP pero de alta mortalidad; sus características clínicas más frecuentes fueron: taquicardia materna, dolor abdominal, hipotensión arterial, palidez, distensión abdominal e hipertensión arterial.
\end{abstract}

Palabras clave: Rotura hepática del embarazo; Preeclampsia severa; Características clínicas. (Fuente: DeCS BIREME).

\section{CLINICAL FEATURES OF LIVER RUPTURE RELATED TO SEVERE PREECLAMPSIA AT THE NATIONAL MATERNAL PERINATAL INSTITUTE}

\begin{abstract}
The liver rupture in pregnancy (LRP) is a disease of low incidence but high morbidity and mortality, being a devastating complication in patients with severe preeclampsia (SPE), approximately 200 cases in the world literature and some individual cases in the national literature are reported. Objectives: The main objective was to describe the most frequent clinical features in patients with SPE admitted to the Unidad de Cuidados Intensivos Materna (UCIM) at the Instituto Nacional Materno Perinatal (INMP). Materials and methods: The medical records of patients with the diagnosis of LRP treated in the UCIM in the period between 2004 and 2013 were reviewed, a record of data collection was used and frequency analysis was performed for most common signs and symptoms. Results: $92,6 \%$ of LRP cases (25/26) occurred in SPE; hospital stay was $19,4 \pm 13,24$ days (2-47), the average UCIM stay was $12,3 \pm 10,24$ days (1-45). The mean age was $33 \pm 7,37$ years (18-44). $50 \%(10 / 20)$ of cases occurred in preterm pregnant and $30 \%(6 / 20)$ in puerperium. $65 \%(13 / 20)$ of patients were multiparous. $38,1 \%(8 / 21)$ of the patients died. The most common clinical features were: maternal tachycardia $(61,9 \%)$, abdominal pain $(57,1 \%)$, hypotension $(57,1 \%)$, pallor $(52,4 \%)$, abdominal distension $(47,6 \%)$ and hypertension $(38,1 \%)$. Conclusions: The LRP is a rare complication of the SPE in the INMP with high mortality; the most frequent clinical features were: maternal tachycardia, abdominal pain, hypotension, pallor, abdominal distension and hypertension.
\end{abstract}

Key words: Hepatic rupture of pregnancy; Severe preeclampsia; Clinical characteristics. (Source: MeSH NLM)

\footnotetext{
Médico Intensivista, Unidad de Cuidados Intensivos Materna del Instituto Nacional Materno Perinatal, Lima - Perú.

Magister en Fisiología Humana.

Magíster en Gerencia de Servicios de Salud

Docente de Postgrado, especialidad de Emergencia de la Facultad de Enfermería de la Universidad Peruana Cayetano Heredia.

Docente de Postgrado, especialidad de Enfermería Intensiva de la Universidad Peruana Cayetano Heredia.
} 


\section{INTRODUCCIÓN}

La rotura hepática del embarazo (RHE) es una complicación infrecuente de la gestación $(0,4$ a 2,2 cada 100000 embarazos) $)^{1,2}$, habitualmente se relaciona con la preeclampsia severa (PES) y, sobre todo, con el síndrome HELLP (acrónimo de Hemolytic anemia, Elevated Liver enzime y Low Platelet count $)^{3-6}$. Fue descrita por primera vez por Abercrombie en $1844^{7,8}$; es un diagnóstico anatomopatológico, se establece en forma macroscópica durante la cirugía o mediante estudios de imágenes, en el preoperatorio inmediato ${ }^{2,9,10}$.

Menos del $5 \%$ de pacientes con síndrome HELLP presentan hemorragia hepática ${ }^{11,12}$ y 1 de cada 2000 pacientes con PES / eclampsia / síndrome HELLP presentan RHE espontánea ${ }^{13}$; sin embargo, la RHE se caracteriza por una elevada mortalidad, la cual varía entre 10 y $80 \% \quad 5,7,14-16$. La literatura mundial describe un aproximado de 200 casos, siendo necesario un mayor volumen de información ${ }^{17-20}$. Según la oficina de estadística del INMP se han reportado 26 casos de RHE entre 2004 y 2013 , con $38,5 \%$ de letalidad y una alta incidencia de complicaciones que prolongan la estancia hospitalaria.

En el Perú, Pilco \& cols. (2006) $)^{6}$ y Marroquin \& cols. $(2010)^{21}$ han descrito casos individuales de RHE en pacientes con PES y síndrome HELLP, con resultado favorable.

El diagnóstico de RHE es difícil de realizar debido a que los síntomas son comunes e inespecíficos; Rinehart \& cols. (1999), en una revisión sobre 141 casos publicados entre 1960 y 1997, describen que los síntomas y signos más frecuentes fueron: Dolor epigástrico $(70 \%)$, hipertensión $(66 \%)$, shock $(56 \%)$, nausea y vómitos $(25 \%)$, dolor de hombro (21\%) y cefalea (11\%); describen una mortalidad materna de $51 \%$ (fetal de $32 \%$ ); también mencionan que más de $75 \%$ de las pacientes tenían síndrome HELLP 22 .

Reck \& cols. (2002), a propósito de 4 casos de RHE en Alemania, publicaron una revisión en la cual mencionan 49 casos publicados en MEDLINE en el período 1990 a 1999; la mortalidad de las pacientes con RHE y hemorragia fue del 39\% (19/49); no describen la frecuencia de signos y síntomas ${ }^{23}$. Araujo \& cols. (2006), en Brasil, describen su experiencia sobre 10 casos de RHE, mencionan que los síntomas más frecuentes fueron sangrado vaginal $(8 / 10)$ y dolor abdominal (7/10); la edad media fue de 42,5 años, la paridad media de 4,5 y la edad gestacional media al finalizar la gestación fue de 35,5 semanas ${ }^{24}$.

Grand'Maison \& cols. (2012) publicaron su experiencia sobre 9 casos de RHE en Canadá ${ }^{7}$, describen que los síntomas más frecuentes fueron dolor epigástrico (6/9) y dolor toráxico o en hombro derecho (5/9); también hacen una revisión de la literatura sobre 93 casos reportados entre 2000 y 2010 , mencionan que $57 \%$ de las mujeres eran multíparas y $92 \%$ tenían PES; la edad media materna era de 32 años. Vigil - De Gracia \& Ortega - Paz (2012) publicaron una revisión sobre PES / eclampsia y $\mathrm{RHE}^{13}$; identificaron 180 casos de rotura / hematoma hepático en el período 1990 - 2010 en 116 publicaciones; 18 con hematoma subcapsular sin RHE y 162 con rotura capsular; la edad media fue de 30,9 $\pm 5,0$ años, $74 / 129(57,4 \%)$ de las mujeres eran multíparas, y se realizó cesárea en $132 / 162(81,5 \%)$ casos. La mortalidad materna fue de $22.2 \%$ durante los 21 años; pero disminuyó a $16,4 \%$ en la última década del estudio. La mortalidad perinatal fue de $30,7 \%$ y se mantuvo muy similar en las dos décadas. Identificaron síndrome HELLP en $92,8 \%$ de los casos.

La presentación clínica del hematoma subcapsular hepático no es característica y ello, aunado a la escasa frecuencia de casos, lleva a que muchas veces no exista la sospecha y el diagnóstico sea demorado y un hallazgo en la laparotomía ${ }^{6,8,25}$. Henny \& cols. (1983; citado por Belfort, 2010) describen una secuencia bifásica durante la rotura de un hematoma subcapsular; los síntomas iniciales son: dolor epigástrico o en cuadrante superior derecho que es constante y de progresivo empeoramiento, con o sin nausea y vómitos. La segunda fase muestra colapso cardiovascular, shock y óbito fetal ${ }^{9}$. También se describe la aparición de síntomas sin alteraciones de laboratorio ${ }^{13}$.

Los síntomas y signos ${ }^{7,21,24,26,27}$ más frecuentes son los siguientes:

1) Dolor abdominal: habitualmente en hipocondrio derecho o epigastrio, el cual puede irradiarse a la espalda 8,27,28. También puede estar ausente como síntoma inicial ${ }^{23}$.

2) Shock, taquicardia materna y fetal, hipertensión, nausea y/o vómitos $3,9,23,27,29$.

3) Agitación, distensión abdominal y signos de irritación peritoneal ${ }^{8,24,28,30}$.

4) Dolor de hombro en solo $20,5 \%$ de casos; parece ser, sin embargo, más específico ${ }^{17}$.

5) Otros síntomas menos frecuentes son la fiebre y el íleo ${ }^{31}$.

Otras causas de hemorragia intrahepática incluyen trauma, carcinoma hepatocelular, hemangioma, adenoma hepático, absceso hepático, hígado graso agudo del embarazo y abuso de cocaína $8,14,16,26$; un $95 \%$ de los casos se relaciona con PES ${ }^{8,17}$. Otras entidades a considerar en el diagnóstico diferencial son aquellas que producen dolor abdominal (colecistitis, pancreatitis, gastroenteritis, entre otras) ${ }^{8,23}$.

La formación de un hematoma subcapsular hepático es más frecuente en el tercer trimestre, aunque también puede ocurrir durante el parto o el puerperio ${ }^{8}$; un tercio de los casos se presenta en el postparto ${ }^{23}$; la RHE puede ser secundaria a cualquier causa que eleve la presión intrabdominal, como el trauma, la transportación, los vómitos, la palpación abdominal, las contracciones del parto o las convulsiones de la eclampsia ${ }^{8}$. 
La RHE es una complicación infrecuente de la gestación pero de alta mortalidad, es necesario contar con información que facilite el diagnóstico oportuno. El estudio tiene por objeto describir las características clínicas más relevantes de la RHE relacionada con la PES en pacientes del INMP; no existen series de casos, en el Perú, que ilustren sobre estas características, los datos proporcionados permitirán establecer una frecuencia de presentación que será de utilidad para el reconocimiento temprano de la RHE.

El objetivo del presente trabajo es identificar las características clínicas de la RHE relacionada con la PES en el INMP.

\section{MATERIAL Y MÉTODOS}

El presente es un estudio descriptivo y retrospectivo.

Participantes. La población de estudio estuvo conformada por todas las pacientes hospitalizadas en el área de UCIM del INMP entre los años 2004 y 2013 que cumplieron con los criterios de inclusión y que presentaron el diagnóstico de RHE, localizamos en total 26 pacientes.

Criterios de Inclusión. 1) Edad de 18 a más años (pacientes adultas). 2) Diagnóstico de Preeclampsia Severa, establecido en base a la historia clínica según los criterios del Task Force on Hypertension in Pregnancy (2013). 3) Ausencia de enfermedades crónicas preexistentes. 4) Diagnóstico clínico o patológico de Rotura Hepática del Embarazo. 5) Hospitalizadas entre el año 2004 y el 2013.

Criterios de exclusión. 1) Rotura hepática relacionada con otras causas: Por ejemplo, traumatismos, tumores, etc. 2) Ausencia de información clínica completa por carencias de archivo u hojas de referencia incompletas.

Variables de estudio. Para todos los signos y síntomas, se tomó en cuenta el momento en que se estableció el diagnóstico presuntivo de RHE.

Instrumentos. Ficha de recolección de datos, la cual consta de tres secciones: Datos generales, datos clínicos y observaciones.

Procedimiento. Se seleccionaron los casos mediante revisión del libro de ingresos del servicio de UCIM, del archivo de certificados de defunción y de los datos proporcionados por la Oficina de Estadística del INMP. Se recolectaron los datos mediante una ficha ad hoc, previa revisión de historias clínicas. Los datos y cuadros estadísticos se procesaron con los programas Excel Office 2010 y SPSS 21. Para la descripción de resultados se emplearon medidas de frecuencia tales como la media y la mediana; para variables categóricas se utilizó la proporción y el porcentaje.

\section{RESULTADOS}

Se reportaron 26 casos de RHE en el período de estudio; $25(96,2 \%)$ correspondían a PES; un caso se excluyó por corresponder a cáncer hepático. Excluimos otros 4 casos por no disponer de historia clínica en dos de ellos y en otros dos por carecer de hojas de referencia completas. Por tanto, los resultados corresponden a 21 pacientes.

La distribución por años se presenta en el gráfico 1.

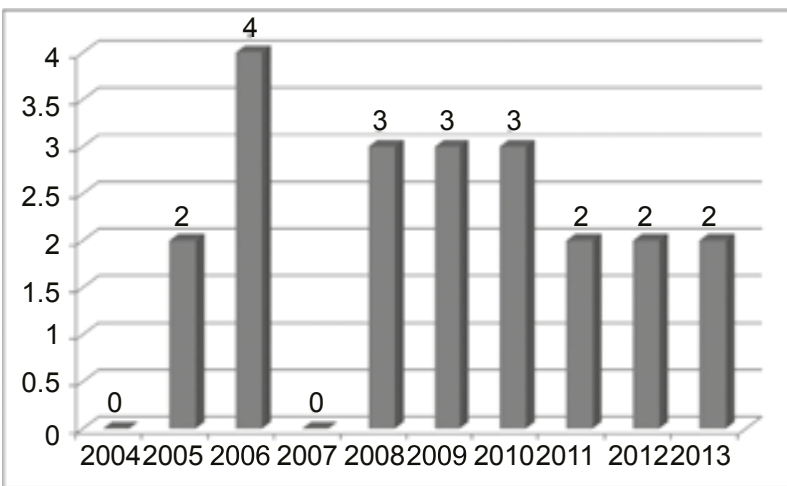

Gráfico 1. Número de casos de rotura hepática del embarazo relacionados con preeclampsia severa por año.

La estancia hospitalaria promedio fue de 19,4 \pm 13,24 días; la estancia promedio en UCI Materna fue de 12,3 \pm 10,24 días (Rango $1-45$ ). La edad promedio fue de 33 $\pm 7,37$ años; la tabla 1 muestra la información sobre los grupos etáreos de las pacientes.

Tabla 1. Distribución según grupos etáreos

\begin{tabular}{ccc}
\hline Grupos etáreos & Frecuencia & Porcentaje \\
\hline > a 35 & 9 & 42,9 \\
18 a 24 & 3 & 14,3 \\
25 a 35 & 9 & 42,9 \\
Total & 21 & 100,0 \\
\hline
\end{tabular}

Al considerar la edad gestacional al momento del diagnóstico de RHE, se observó un $50 \%$ (10 / 20) de casos pretérmino y un $30 \%(6 / 10)$ de casos en el puerperio. La paridad se muestra en la tabla 2 .

Tabla 2. Distribución de las pacientes con diagnóstico de rotura hepática del embarazo según paridad previa.

\begin{tabular}{ccc}
\hline PARIDAD & Frecuencia & Porcentaje \\
\hline Multípara & 13 & 65,0 \\
Nulípara & 7 & 35,0 \\
Total & 20 & 100,0 \\
\hline
\end{tabular}

Del total de pacientes consideradas en el estudio, fallecieron $8(38,1 \%)$ y 13 recibieron el alta en condición de mejoradas $(61,9 \%)$.

La ubicación de la rotura hepática según reporte quirúrgico se presenta en el gráfico 2 . 


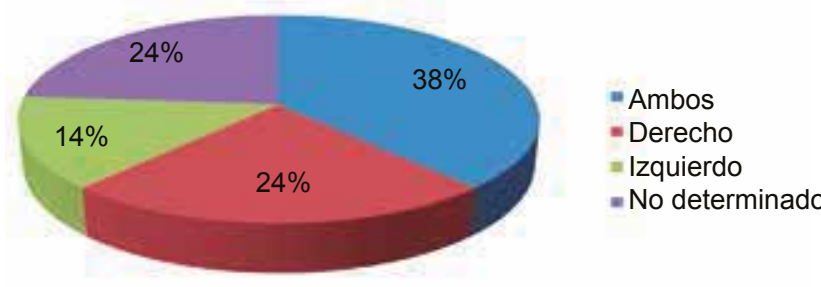

Gráfico 2. Ubicación de la rotura hepática según lóbulos comprometidos.

La tabla 3 muestra la frecuencia de los signos y síntomas establecidos como variables. La presencia de dolor abdominal sube hasta el $75 \%$ en pacientes sin sedación. La distensión abdominal se presentó en $100 \%$ de las pacientes con sedación. Debe destacarse que un número significativo de pacientes recibían sedación al momento que se estableció el diagnóstico (5 de 21 casos).

Tabla 3. Características clínicas de la rotura hepática del embarazo.

\begin{tabular}{ccc}
\hline Signo o síntoma & Número & Porcentaje \\
\hline Taquicardia materna & 13 & 61,9 \\
\hline Dolor abdominal & 12 & 57,1 \\
\hline Hipotensión arterial & 12 & 57,1 \\
\hline Distensión abdominal & 10 & 47,6 \\
\hline Hipertensión arterial & 8 & 38,1 \\
\hline Nausea y vómitos & 4 & 19,1 \\
\hline Desvanecimiento & 1 & 4,8 \\
\hline Taquicardia fetal & 1 & 4,8 \\
\hline
\end{tabular}

Respecto a la ubicación del dolor abdominal, síntoma presente en 12 pacientes, un tercio de los casos era de localización difusa; dos tercios se localizaban en epigastrio o hipocondrio derecho; el gráfico 3 muestra la ubicación del dolor.

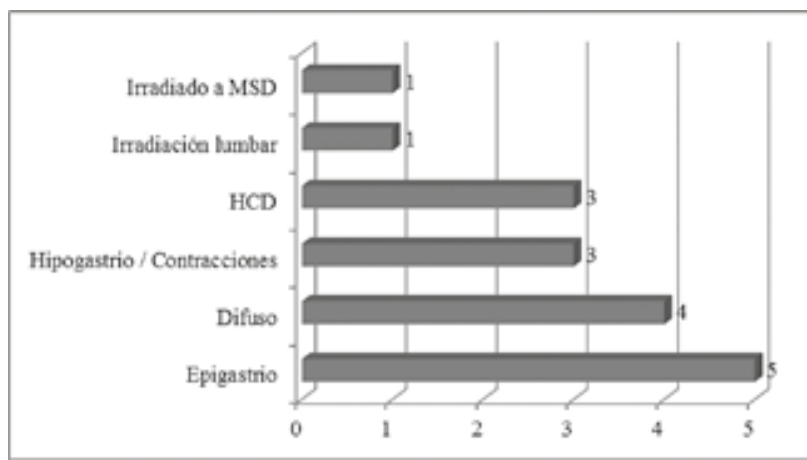

Gráfico 3. Ubicación del dolor abdominal.

En nuestra serie de casos, 18 de las 21 pacientes presentaban otros síntomas y signos al momento del diagnóstico, el gráfico 4 condensa esa información.

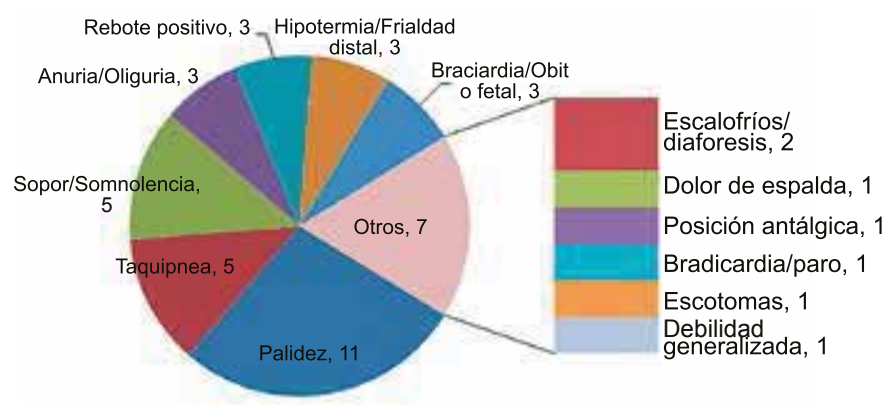

Gráfico 4. Otros síntomas y signos.

\section{DISCUSIÓN}

El $96,2 \%$ de nuestros casos de RHE correspondió a mujeres con PES. Grand'Maison \& cols. (2012), en una revisión sobre 93 casos $^{7}$, reportan la presencia de PES en 59 de 64 casos (92\%); es probable que esta frecuencia sea aún mayor al aplicar los criterios establecidos por el Task Force on Hypertension in Pregnancy ${ }^{32}$ en 2013, en el cual se excluye a la proteinuria como criterio obligatorio para el diagnóstico de PES.

La estancia hospitalaria promedio fue de 19,4 días; la estancia promedio en UCl Materna fue de 12,3 días. Grand'Maison et al (2012) en su experiencia sobre 9 casos en Canadá ${ }^{7}$, refieren que la estancia promedio fue de 10 días (Rango 5 a 51). Araujo \& cols (2006) en su experiencia sobre 10 casos en Brasil ${ }^{24}$, mencionan una estancia de $14 \pm 13,2$ días. Seis de las 9 pacientes del grupo canadiense fueron tratados mediante embolización selectiva, un procedimiento que reduce la estancia hospitalaria y la mortalidad ${ }^{13,27,33}$. El INMP no cuenta con la posibilidad de realizar procedimientos de embolización y el tratamiento habitual para la RHE es el taponamiento hepático y la laparostomía.

Se observó un número significativo de mujeres añosas (42,9\%), lo cual guarda semejanza con los hallazgos de Araujo \& cols. (2006), que describen una media de 42,5 años para 10 casos $^{24}$; la edad media de nuestras pacientes fue de 33 años, similar a la reportada por Grand'Maison et al (2012), sobre 93 casos (media de 32 años) ${ }^{7}$, y de Vigil De Gracia \& Ortega - Paz (2012), sobre 163 casos (media de $30,9 \pm 5$ años) ${ }^{13}$. Podemos afirmar que la RHE es una complicación que se presenta con mayor frecuencia en mujeres con más de 30 años de edad y, sobre todo, en el grupo de gestantes añosas.

La edad gestacional (EG) de nuestro grupo de estudio fue catalogada como pretérmino en $47,6 \%$ de los casos, habiéndose presentado la RHE durante el puerperio en $28,6 \%$ de los casos; Araujo \& cols. (2006) describen la EG media de 35,5 semanas $^{24}$. Vigil - De Gracia \& Ortega - Paz ${ }^{13}$ mencionan una EG media de 33,2 $\pm 4,9$, sobre 149 pacientes. Podemos afirmar que la RHE es más frecuente en gestantes pretérmino, lo cual es congruente con el hecho de que la PES se presenta en forma más 
Tabla 4. Tasa de mortalidad materna según diferentes estudios.

\begin{tabular}{cccc}
\hline Autores y año & Tipo de estudio (referencia) & $\begin{array}{c}\text { Número de } \\
\text { Casos }\end{array}$ & Tasa de mortalidad materna \\
\hline Rinehart \& cols., 1999 & Revisión $^{22}$ & 141 & $32 \%$ \\
Reck \& cols., 2002 & Revisión $^{23}$ & 49 & $39 \%$ \\
Araujo \& cols., 2006 & Serie de casos ${ }^{24}$ & $10 \%$ \\
Grand'Maison \& cols., 2012 & Serie de casos ${ }^{7}$ & 10 & $0 \%$ \\
Grand'Maison \& cols., 2012 & Revisión $^{7}$ & 9 & $17 \%$ \\
Vigil - De Gracia \& Ortega - Paz, & Revisión ${ }^{13}$ & 180 & $22,2 \%$ \\
2012 & & $28,9 \%$ en casos de 1990 - 2000. & $16,4 \%$ en casos de 2001-2010. \\
\hline
\end{tabular}

agresiva en ese grupo de mujeres, siendo más frecuente la presentación del síndrome HELLP en gestantes con EG menor a 35 semanas ${ }^{9,34}$. Un alto porcentaje de casos se presentó en el puerperio, lo cual reafirma la necesidad de vigilancia en ese grupo de pacientes.

Predominaron en nuestro estudio las multíparas $(61,9 \%)$, un hallazgo similar es reportado por Grand'Maison \& cols ${ }^{7}$, con $57 \%$ y por Vigil - De Gracia \& Ortega - Paz ${ }^{13}$, con un $57,4 \%$ (74/129); estos datos inducen a considerar que la multiparidad es un factor de riesgo para RHE en pacientes con PES, siendo mayor el riesgo en gestantes añosas.

Un $38,1 \%$ de nuestras pacientes fallecieron; la mortalidad materna descrita por otros estudios se muestra en la tabla 4. Se observa una tendencia a la disminución de la mortalidad a lo largo de los años en los estudios de revisión de casos; en la publicación de Vigil - De Gracia (2012) la mortalidad se redujo de $28,9 \%$ en los noventa a $16,4 \%$ en el período 2000 - 2010; la mayor parte de los casos incluidos en estos estudios procede de países de habla inglesa, en la mayoría de los cuales ya se han introducido técnicas como la embolización selectiva, el transplante hepático y el uso de factor VII activado, medidas que han incidido sobre la reducción de la morbimortalidad y una menor estancia hospitalaria ${ }^{7,12,23}$. Es de recalcar que la tasa de mortalidad materna ${ }^{13}$ antes de 1970 se aproximaba al $100 \%$.

Respecto a la ubicación de la RHE, encontramos compromiso del lóbulo derecho en $24 \%$ de casos y del lóbulo izquierdo en 14\%; estos porcentajes suben al 62 y $52 \%$, respectivamente, al considerar los casos con compromiso bilobar. Vigil - De Gracia \& Ortega - Paz
$(2012)^{13}$, mencionan que $77 \%$ de los casos (77/100) incluidos en su revisión presentaban compromiso del lóbulo derecho. El compromiso del lóbulo derecho es más frecuente en todas las series de $\operatorname{casos}^{35}$. Todos nuestros casos fueron diagnosticados mediante visualización directa en la cirugía, mientras que en los estudios de revisión se mencionan numerosos casos en los que el diagnóstico se hizo mediante estudios de imagen 7,13 .

Al comparar la frecuencia de las características clínicas según nuestro estudio y la publicada en estudios de revisión y series de casos, obtuvimos la tabla 5 . El dolor abdominal, presente en $57,1 \%$ de nuestros casos, asciende a $75 \%$ cuando se considera únicamente a las pacientes sin sedación (12/16). Este síntoma aparece en todas las series publicadas, aunque debe tenerse en cuenta que muchas pacientes con PES reciben sedación debido a otras complicaciones de la enfermedad ${ }^{9,34}$; este hecho dificulta el diagnóstico y debe tenerse presente al momento de considerar el diagnóstico de RHE durante el puerperio inmediato. El dolor abdominal es particularmente útil para el diagnóstico de RHE puesto que su presencia, sobre todo cuando se ubica en epigastrio o hipocondrio derecho, permite diferenciar este cuadro de otras causas de shock hemorrágico. La ubicación predominante del dolor en epigastrio (5 de 12 casos), aunada al dolor en hipocondrio derecho (3/12 casos) concuerda con la bibliografía mundial, que reporta presencia de dolor epigástrico en $70 \%$ (28) y $66,7 \%$ de casos ${ }^{7}$. La irradiación del dolor hacia región lumbar y miembro superior derecho se presentó en una sola paciente para cada caso; sin embargo la irritación diafragmática con el consecuente estímulo del nervio frénico justifica, desde el punto de vista

Tabla 5. Características clínicas de la rotura hepática del embarazo según diversos estudios.

\begin{tabular}{|c|c|c|c|c|}
\hline & $\begin{array}{c}\text { Rinehart \& } \text { cols }^{22} \text {, } \\
1999\end{array}$ & $\begin{array}{c}\text { Araujo \& } \text { cols }^{24} \text {, } \\
2006\end{array}$ & $\begin{array}{c}\text { Grand'Maison \& } \\
\text { Cols }^{7}, 2012 \\
\end{array}$ & $\begin{array}{c}\text { Estudio } \\
\text { presente }\end{array}$ \\
\hline Tipo de estudio & Revisión & Serie de casos & Serie de casos & Serie de casos \\
\hline Casos & 141 & 10 & 9 & 21 \\
\hline Taquicardia materna & & & & $61,9 \%$ \\
\hline Dolor abdominal & $70 \%$ (epigastralgia) & $70 \%$ & $66,7 \%$ (epigastralgia) & $57,1 \%$ \\
\hline Hipotensión arterial & $56 \%$ & & & $57,1 \%$ \\
\hline Distensión abdominal & & & & $47,6 \%$ \\
\hline Hipertensión arterial & $66 \%$ & & & $38,1 \%$ \\
\hline Nausea y vómitos & $25 \%$ & & & $19,1 \%$ \\
\hline Dolor de hombro & $21 \%$ & & $55,6 \%$, incluye dolor toráxico. & \\
\hline Cefalea & $11 \%$ & & & \\
\hline Sangrado vaginal & & $80 \%$ & & \\
\hline
\end{tabular}


fisiopatológico, la presencia de estos síntomas dentro del cuadro clínico de la RHE.

Los trastornos hemodinámicos, expresados por taquicardia materna $(61,9 \%)$ e hipotensión arterial $(57,1 \%)$ son signos frecuentes en pacientes con RHE, Rinehart \& cols. reportaron la presencia de hipotensión en $56 \%$ de casos $^{22}$; sin embargo pueden presentarse en otras situaciones de hemorragia periparto, tales como la atonía uterina, los desgarros cervico - vaginales y hemorragias intraabdominales de otro origen ${ }^{2,9,34}$; consideramos que su valor es importante al establecer la posibilidad clínica de una hemorragia significativa en pacientes con factores de riesgo para el sangrado masivo como son las puérperas inmediatas o las gestantes del tercer trimestre con PES.

La distensión abdominal se presentó en $100 \%$ de las pacientes con sedación (5/5); su porcentaje desciende a $31,6 \%$ en pacientes sin sedación $(5 / 16)$; estos hechos son relevantes en pacientes que no pueden manifestar otros síntomas, tales como nausea, vómito o dolor abdominal, y podría plantearse la presencia de distensión abdominal cuantificada mediante perímetro abdominal como un signo probable de RHE, asignándosele un mayor valor a pacientes con sedación.

La hipertensión arterial, presente en $38,1 \%$ de nuestras pacientes es un signo y criterio diagnóstico de preeclampsia, Rinehart \& cols reportaron su presencia en $66 \%$ de casos $^{22}$; la caída de la presión arterial en pacientes previamente hipertensas es particularmente útil para la sospecha de hemorragias significativas durante el puerperio; en el estudio de Rinehart \& cols. se menciona la presencia de hipertensión arterial en $66 \%$ de casos y de hipotensión arterial en $56 \%$ de casos ${ }^{22}$, lo que implica que un número significativo de pacientes presentaron caída brusca de la presión arterial durante el desarrollo de la RHE.

La nausea y el vómito, presentes en $19,1 \%$ de nuestros casos, se aproximan al valor de $25 \%$ publicado por Rinehart \& cols (1999) $)^{22}$; se describen como síntomas propios de la RHE, sin embargo su presencia en menos de la cuarta parte de las pacientes y la diversidad de patologías en las que se presentan obligan a una valoración clínica cuidadosa. El desvanecimiento y la taquicardia fetal se presentaron, cada uno, solo en un caso; sin embargo la literatura mundial resalta su importancia como signos de trastornos hemodinámicos en mujeres gestantes ${ }^{9}$. También identificamos otros signos y síntomas dentro de la presentación inicial de la RHE, muchos de ellos correlacionan con diversos estadíos del shock hemorrágico (Foley, 2012) ${ }^{34}$; destacan los siguientes:

- Palidez, presente en $11 / 21$ casos; es un signo inespecífico de trastornos hemorrágicos.

- Taquipnea, presente en 5 / 21 casos, es un signo de shock hemorrágico estadío $\|^{33}$.

- Sopor y somnolencia, presentes en 5 / 21 casos, se presentan en el shock hemorrágico de estadío II y III ${ }^{7,13}$, por lo que representan signos de alarma.

- Oliguria y anuria, presentes en 3 / 21 casos, también son signos de shock hemorrágico, correspondientes a los estadíos III y IV, respectivamente ${ }^{7}$; son signos de alarma.

- Rebote positivo, presente en 3 / 21 pacientes; es un signo clínico que requiere la palpación enérgica del abdomen, maniobra que debe evitarse en sospecha de RHE.

- La hipotermia y frialdad distal, presentes en $3 / 21$ pacientes, también son signos de shock hemorrágico estadio III y IV ${ }^{34}$ e implican hipoperfusión distal.

- La bradicardia y el óbito fetal, presentes en 3/21 casos, se presentan como signos exclusivos de pacientes gestantes con complicaciones hemodinámicas ${ }^{9}$; debe recordarse que el útero es un órgano pobremente perfundido en estados de shock, por lo que el compromiso fetal es un signo de utilidad para el diagnóstico de shock materno.

- El escalofrío, la diaforesis y la debilidad generalizada, así como el paro cardiorespiratorio, corresponden a diferentes estadíos de shock hemorrágico ${ }^{9,34}$; el dolor en dorso y la posición antálgica corresponden a diferentes manifestaciones del estímulo doloroso originado en la cápsula de Glisson y en el área subdiafragmática.

- Una sola paciente refirió presencia de escotomas (1/21), debe recordarse que $5 / 21$ casos recibían sedación al momento del diagnóstico de RHE, hecho que dificulta la valoración.

Las limitaciones de nuestro estudio fueron las siguientes: a) Al ser un estudio unicéntrico, las conclusiones deben extrapolarse con precaución a la población general. b) Se excluyeron cuatro pacientes del estudio debido a carencia de la historia clínica o a hojas de referencia incompletas; se requiere un mejor manejo de la información clínica tanto en los centros emisores como receptores de las pacientes transferidas.

Las conclusiones más relevantes del estudio fueron:

- Las características clínicas relacionadas a RHE más frecuentes fueron: taquicardia materna $(61,9 \%)$, dolor abdominal $(57,1 \%)$, hipotensión arterial $(57,1 \%)$, distensión abdominal $(47,6 \%)$, hipertensión arterial $(38,1 \%)$ y nausea y vómitos $(19,1 \%)$.

> El dolor abdominal se presentó en $75 \%$ de pacientes sin sedación (12/16).

> La distensión abdominal se presentó en 100\% (5 / 5) de las pacientes con sedación.

, Dieciocho de las 21 pacientes presentaron otros síntomas y signos, tales como: Palidez $(52,4 \%)$, taquipnea $(23,8 \%)$, sopor / somnolencia $(23,8 \%)$, anuria /oliguria $(14,3 \%)$, rebote positivo $(14,3 \%)$, 
hipotermia /frialdad distal (14,3\%), bradicardia /óbito fetal $(14,3 \%)$.

, Las características clínico - epidemiológicas del grupo estudiado fueron: El 92,6\% (25/26) de los casos se presentó en pacientes con PES. La estancia hospitalaria fue de 19,4 \pm 13,24 días (Rango 2 - 47); la estancia en UCl Materna fue de 12,3 \pm 10,24 días (Rango 1 - 45). La edad promedio fue de $33 \pm 7,37$ años (Rango 18 a 44). Nueve de 21 pacientes eran añosas (edad mayor a 35 años), lo que representa un $42,9 \%$. Un $50 \%$ (10 120) de los casos de RHE se presentó en gestantes pretérmino y un $30 \%(6 / 20)$ en puerperas. El $65 \%(13 / 20)$ de las pacientes eran multíparas. El $38,1 \%(8 / 21)$ de las pacientes fallecieron.

, La RHE afectó en forma predominante a ambos lóbulos hepáticos (38\%).

\section{REFERENCIAS BIBLIOGRÁFICAS}

1. Alassia M, Buteler MM, et al. Hematoma hepático en el síndrome HELLP. Revista de la Facultad de Ciencias Médicas, 2011; 68(3): 119 - 124.

2. Malvino, E. Causas infrecuentes de hemorragias abdominopelvianas en el embarazo. En: Shock hemorrágico en obstetricia. Malvino, E (ed.) Buenos Aires, 2010. Pre - edición de distribución gratuita (pp $41-56$ ).

3. Gutiérrez CE et al. Letters to the Editor. Hepatic rupture and hemoperitoneum in a pregnant woman with HELLP sindrome. Rev Esp Enferm Dig, 2010; 102 (7): 453 - 454.

4. Kulungowski AM, et al. Hemolysis, elevated liver enzymes, and low platelets syndrome: when is surgical help needed? The American Journal of Surg, 2009; 198: 916 - 920.

5. Miguelote RF, et al. Postpartum spontaneous rupture of a liver hematoma associated with preeclampsia and HELLP síndrome. Arch Gynecol Obstet,2009; 279: 923- 926.

6. Pilco P, et al. Hematoma hepático subcapsular roto en Sindrome Hellp. Revisión de la literatura y reporte de un caso con manejo conservador. Rev Gastroenterol Perú, 2006; 26: 207 - 210.

7. Grand'Maison S, Sauvé N, et al. Hepatic rupture in Hemolysis, Elevated Liver Enzymes, Low Platelets syndrome. Obstet Gynecol,2012; 119: 617-625.

8. Pérez M \& Sáez V. Hematoma subcapsular hepático. Grave complicación del embarazo. Rev Elect. de las Ciencias Médicas en Cienfuegos. Medisur, 2010; 8(6): 445 - 451.

9. Roberts, S. Hypovolemic and Cardiac Shock. En: Critical care obstetrics. Belfort, M (ed). Ed. Wiley - Blackwell, Chichester, West Sussex, RU, 2010 (pp. 559-564).

10. Borrás $D$, Fortuño $S$ et al. Rotura hepática asociada a preeclampsia y síndrome HELLP, con resultados catastróficos. Prog Obstet Ginecol.,2009; 52(7): $402-6$.

11. Darby $M$ et al. Using case reports to determine when liver bleeding occurs during disease progression in HELLP síndrome. International Journal of Gynecol and Obstet, 2013; 123: 7 - 9.

12. Sheikh $R$, Yasmeen $S$, et al. Spontaneous Intrahepatic Hemorrhage and Hepatic Rupture in the HELLP Syndrome: Four Cases and a Review. Journal of Clinical Gastroenterology, 1999; Volume 28(4): 323 - 328.

13. Vigil - De Gracia P \& Ortega-Paz L. Pre-eclampsia / eclampsia and hepatic rupture. International Joumal of Gynecology and Obstetrics, 2012; 118: 186 - 189.
14. Joshi D, James A, Quaglia A, Westbrook RH \& Heneghan MA. Liver disease in pregnancy. Lancet, 2010; 375: $594-605$.

15. Martínez A, Arvizu R, et al. Rotura hepática espontánea en el síndrome HELLP: tratamiento mediante packing. Prog Obstet Ginecol., 2010; 53(5): $194-197$

16. Singh Y, Kochar, SPS, et al. Hepatic Rupture Complicating HELLP Syndrome in Pregnancy. Medical Journal Armed Forces India, 2009; Vol. 65, No. 1: 89 - 90.

17. Holst B, McGuinness E \& Morris - Stiff G. Spontaneous peripartum liver haemorrhage presenting as foetal distress. Grand Rounds, 2013; Vol 13: 42 - 46.

18. Kelly $\mathrm{J}$ et al. Second trimester hepatic rupture in a 35 year old nulliparous woman with HELLP syndrome: a case report. World Journal of Emergency Surg, 2009; 4: 23 - 26.

19. Matheï J, Janssen A, et al. Spontaneous Postpartum Subcapsular Liver Rupture. Acta chir belg, 2007; 107: 713 - 715

20. Poo JL, Gónfora J. Symposium liver \& pregnancy hepatic hematoma and hepatic rupture in pregnancy. Annals of Hepatology 2006; 5 (3): $224-226$.

21. Marroquín $P A$, Cabrera $S$, Gutiérrez $L$ \& Hijar $A$. Rotura hepática en paciente con preeclampsia severa y síndrome HELLP. Rev Per Ginecol Obstet.,2010; 56: 310 - 312.

22. Rinehart BK, et al. Preeclampsia - associated hepatic hemorrhage and rupture: mode of management related to maternal and perinatal outcome. Obs Gyn Surv, 1999; 54: 196-202.

23. Reck T, Bussenius - Kammerer M, et al. Tratamiento quirúrgico de la rotura hepática asociada con el síndrome HELLP - actualización. European Journal of Obstetrics \& Gynecology and Reproductive Biology (Ed. Española), 2002; 2: 24 - 32.

24. Araujo AC, Leao MD, et al. Characteristics and treatment of hepatic rupture caused by HELLP syndrome. Am J Obstet Gynecol, 2006; 195: $129-33$.

25. Ouviña O, Alonso Vaquero MJA et al. Hematoma subcapsular hepático en el síndrome de HELLP. Prog Obstet Ginecol., 2010; 53(9): 373 - 376.

26. Peñaflores - Rodríguez et al. Síndrome de HELLP y hematoma subcapsular hepático. Reporte de un caso. Salud en Tabasco, 2007; Vol. 13, No. 1:597 - 601.

27. Pliego A et al. Rotura hepática espontánea durante el embarazo. Serie de cuatro casos y revisión de la literatura médica. Ginecol Obstet Mex,2006; 74: 224 - 231.

28. Arias M, Prieto MA, et al. Rotura espontánea hepática en el Síndrome HELLP. MEDICRIT Revista de Medicina Interna y Medicina Crítica, 2006; 3(1): 22 - 25.

29. Al-Othebi KHA, et al. Unforgettable lesson: Emergency in Emergency Room. Research Journal of Obstetrics and Gynecology,2012; 5 (1): 8 - 12.

30. Barton JR \& Sibai BM. Gastrointestinal Complications of Preeclampsia. Semin Perinatol, 2009; 33: 179 - 188.

31. Zetterman RK. Liver Disease in Pregnancy. Medscape Gastroenterology, 2013. En medscape.com/viewarticle/781889. Leído el 15 de octubre de 2013

32. Task Force on Hypertension in Pregnancy. En: Hypertension in pregnancy. American College of Obstetrician and Gynecologists, Washington, 2013.

33. You JS, Chung YE, et al. Spontaneous hepatic rupture caused by hemolysis, elevated liver enzymes, and low platelet count síndrome. Am J Emerg Med (en prensa), En http://dx.doi. org/10.1016/j.ajem.2013.12.001. Leído el 15 de enero de 2014.

34. Sibai, BM. Emergencias hipertensivas. En: Cuidados intensivos en obstetricia. Foley, M. (ed.). Ed. Amolca, Caracas, 2012 (pp. 49-60).

35. Revilla Rodríguez $\mathrm{E}$, et al. Caso clínico. Ruptura hepática en una paciente con preeclampsia y ligadura de arteria hepática común. Reporte de un caso. Revista de la Asociación Mexicana de Medicina Crítica y Terapia Intensiva, 2008; Vol. 22, Núm. 4: 254 - 259. 